\title{
Pillow sign in colonoscopy
}

\begin{abstract}
Colonic lipomas are benign adipose colonic mesenchymal tumor. They are usually asymptomatic and usually diagnosed as "pillow sign" in either screening or diagnostic colonoscopy. Pillow sign is defined as indentation of colonic tumors with the use of the biopsy forceps during colonoscopy. Here, we report a 62 -year- old -asymptomatic female with pillow sign in screening colonoscopy.
\end{abstract}

Keywords: pillow sign, colonic lipoma, benign large lipoma of large bowel
Volume 8 Issue 2 - 2018

\author{
Lin, Kyawzaw,' Ofori ,Emmanuel, ${ }^{2}$ \\ Linn,Sandar, ${ }^{2}$ Febin John, ${ }^{2}$ Lin Aung Naing,' \\ Reddy Madhavi, ${ }^{3}$ \\ 'Internal Medicine Department, The Brooklyn Hospital Center, \\ Affiliate of the Mount Sinai Hospital, USA \\ ${ }^{2}$ Gastroenterologist Fellow, The Brooklyn Hospital Center, \\ Affiliate of the Mount Sinai Hospital, I2I, USA \\ ${ }^{3}$ Gastroenterologist , Attending and Associate Program Director, \\ The Brooklyn Hospital Center,Affiliate of the Mount Sinai \\ Hospital, USA
}

Correspondence: Kyawzaw Lin, Department of Medicine, The Brooklyn Hospital Center, USA, Tel +315-664-1916,

Email dr.kyawzawlin20I5@gmail.com

Received: March 18, 2018 | Published: March 23, 2018

\section{Case report}

A 62 year old female with past medical history of hypertension and osteoarthritis was referred from her primary care physician for age appropriate screening colonoscopy. She denies nausea, vomiting, abdomen pain, fever, weight loss, hematochezia, loss of weight or loss of appetite. She has significant family history of colon cancer in her mother at the age of 80 . Physical examination and routine labs were insignificant. After standardized bowel preparation, she underwent screening colonoscopy. On colonoscopy, a $3 \mathrm{~cm}$ lipomatous lesion was seen in the ascending colon with a positive "pillow sign". ${ }^{1}$ Multiple diverticuli are found in the descending colon and sigmoid colon.

\section{Conclusion}

Colonic lipomas are accidentally found during diagnostic or screening colonoscopy. They are usually asymptomatic but can sometimes present with complications such as vague abdomen pain, bleeding per rectum, intestinal obstruction, or intussusception particularly in large lipoma larger than $2 \mathrm{~cm}$. Management depends on size and presenting symptoms .However, there is no proper guideline to follow up for those less than $2 \mathrm{~cm}$ asymptomatic colonic lipomas. The size of the stalk is more important than the size of lipoma in considering endoscopic removal.

\section{Discussion}

Colonic lipomas are benign non-epithelial adipocyte hyperplasia of the gastro-intestinal tract. They are the second common begin large bowel tumor after adenomatous polyps. They are mesenchymal lesions of the large bowel; may be single or multiple and broad based or pedunculated. Rarely, they transform to liposarcoma. Almost all the colonic lipomas are found in the submucosa $(90 \%)$ and in a few cases, they are found in sub-serosa and intra-mucosa. The incidence rate varies from $0.2 \%$ to $4.4 \% .{ }^{1}$ In order of decreasing frequency, lipomas are found in large bowel, cecum, ascending colon and sigmoid colon. They are most commonly found in females than males while the ascending colon is the common site in women and the descending colon in men. The median age is between 50 and 70 .

Lipomas are found to be well-defined, soft, oval, yellowish mass with intact mucosa but sometimes with eroded mucosa. Rarely, the mucosa may alter with local changes such as atrophy, hyperplasia, adenomatous changes with ulceration, necrosis mimicking malignancy. They are usually asymptomatic and usually accidentally found during colonoscopy, surgery, autopsy, computed tomography , barium enema and endoscopic ultrasonography. Sometimes, they present with vague symptoms such as abdomen pain (55\%), bleeding per rectum ( $40 \%$ ) resulting from ulcerative lesions, diarrhea, subacute intestinal obstruction, intussusception ( 5-7\%) in case of large colonic lipoma large than $2 \mathrm{~cm}^{2}$

Generally, asymptomatic colonic lipomas less than $2 \mathrm{~cm}$ do not need treatment. However, interventions should be considered in the presence of suspicious features of malignancy such as presenting with intussusception, ill-defined margin, associated mesenteric lymphadenopathy, bowel wall thickening on imaging, presence of fungating, ulcerated or necrotic masses on colonoscopy. Giant colonic lipoma may experience frequent torsion and ischemia resulting in inflammatory changes in surrounding mucosa and found as altered appearances on endoscopy. Rarely, spontaneous explosion of colonic lipoma from auto-amputation from intermittent ischemia has been reported. ${ }^{3}$

The diagnosis of colonic lipoma is done by colonoscopy, surgery, autopsy, computed tomography, barium enema and endoscopic ultrasonography. The pathognomonic findings on colonoscopy include Cushion sign or Pillow sign (tumor indents on depression with biopsy forceps): naked fat sign (finding of extrusion of yellowish fatty materials after repeated biopsies or during colonoscopy) and Tent sign (rise of mucosa over the lipoma with the biopsy forceps). They can be found as a homogenous density of adipose tissue on computed tomography. Endoscopic ultrasound (EUS) can be used to diagnose colonic lipomas. In EUS, lipomas can be seen as a hyperechoic 
lesion in submucosa but often atypical heterogenous or hypoechoic mass can be appeared ${ }^{4}$. EUS can also assist to determine whether the stalk of colonic lipoma contain serosa components or not to avoid complications before the interventions.

Management depends on size and symptomatology of colonic lipoma. There is no proper guideline for the necessity and frequency to follow up for those less than $2 \mathrm{~cm}$ asymptomatic colonic lipomas. Endoscopic resection techniques such as snare resection, endoloop ligation are increasing popularity in treating symptomatic colonic lipomas though there is a risk of perforation particularly gigantic lipomas. Surgical treatments including segmentectomy or hemicolectomy are mostly reserved for large or colonic lipomas associated with complications. In the literature, using snare electrocautery, endoscopic removal of large pedunculated large tumor more than $2 \mathrm{~cm}$ (as large as $11 \mathrm{~cm}$ ) is safe. ${ }^{5}$

Regarding large lipomas, the size of the stalk is the key compared to size of lipoma in considering endoscopic removal. Endoloop ligation of colonic lipomas has a promising trend in management for large lipomas and has been succeeded in removal of lipomas from muscular is propria if the tumors are massive with long stalk. ${ }^{6}$

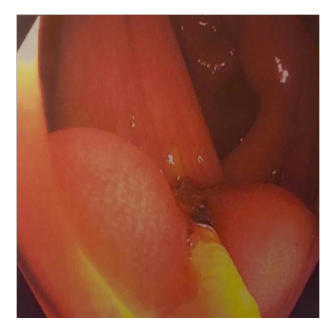

Figure I A $3 \mathrm{~cm}$ lipomatous lesion seen in the ascending colon with a positive "Pillow sign".

\section{Conflict of interests}

The authors declare there is no conflict of interest.

\section{Acknowledgements}

None.

\section{References}

1. Vecchio R, Ferrara M, Mosca F, et al. Lipomas of the large bowel. The European journal of surgery. 1996;162(11):915-9.

2. Bahadursingh AM, Robbins PL, Longo WE. Giant submucosal sigmoid colon lipoma. The American journal of surgery. 2003;186(1):81-2.

3. Gupta AK, Mujoo V. Spontaneous autoamputation and expulsion of intestinal lipoma. J Assoc Physicians India. 2003;51:833.

4. Shepherd BD, Merchant N, Fasig J, et al. Endoscopic ultrasound diagnosis of pelvic lipoma causing neurologic symptoms. Digestive diseases and sciences. 2006;51(8):1364-6.

5. Stone C, Weber HC. Endoscopic removal of colonic lipomas. The American journal of gastroenterology. 2001;96(4):1295.

6. Kaltenbach T, Milkes D, Friedland S, et al. Safe endoscopic treatment of large colonic lipomas using endoscopic looping technique. Digestive and Liver Disease. 2008;40(12):958-61. 